\title{
Structure of incommensurate gold sulfide monolayer on Aul111)
}

\section{Citation}

Quek, Su Ying, Monika M. Biener, Juergen Biener, Joydeep Bhattacharjee, Cynthia M. Friend, Umesh V. Waghmare, and Efthimios Kaxiras. 2007. "Structure of Incommensurate Gold Sulfide Monolayer on Au(111)." The Journal of Chemical Physics 127 (10): 104704. https:// doi.org/10.1063/1.2770731.

\section{Permanent link}

http://nrs.harvard.edu/urn-3:HUL.InstRepos:41384021

\section{Terms of Use}

This article was downloaded from Harvard University's DASH repository, and is made available under the terms and conditions applicable to Other Posted Material, as set forth at http:// nrs.harvard.edu/urn-3:HUL.InstRepos:dash.current.terms-of-use\#LAA

\section{Share Your Story}

The Harvard community has made this article openly available.

Please share how this access benefits you. Submit a story.

Accessibility 


\title{
Structure of incommensurate gold sulfide monolayer on Au(111)
}

\author{
Su Ying Quek ${ }^{1}$, Monika M. Biener ${ }^{1,2}$, Juergen Biener ${ }^{3,6}$, Joydeep Bhattacharjee ${ }^{5}$, \\ Cynthia M. Friend ${ }^{1,2}$, Umesh V. Waghmare ${ }^{5}$ and Efthimios Kaxiras ${ }^{1,4}{ }^{*}$ \\ ${ }^{1}$ Division of Engineering and Applied Sciences, \\ ${ }^{2}$ Department of Chemistry and Chemical Biology, \\ ${ }^{3}$ Center for Nanoscale Science, ${ }^{4}$ Department of Physics \\ Harvard University, Cambridge, Massachusetts 02138-2902, USA \\ ${ }^{5}$ Theoretical Sciences Unit, Jawaharlal Nehru Centre for Advanced Scientific Research, \\ Jakkur PO, Bangalore 560 064, India
}

*Corresponding author. Electronic address: kaxiras@physics.harvard.edu

${ }^{6}$ Current affiliation: Nanoscale Synthesis and Characterization Laboratory, Lawrence Livermore National Laboratory, 7000 East Ave, Livermore, California 94550 USA 


\begin{abstract}
Two-dimensional confined systems, such as substrate-supported incommensurate layers, are of interest because their structural and electronic properties may differ from those of bulk materials. While advances in experimental techniques have resulted in the growth of many such interesting systems, progress can often be hampered by the lack of an atomistic-scale understanding of the structure, especially for incommensurate systems. In this work, we develop an atomic-scale model for an ordered incommensurate gold-sulfide (AuS) adlayer that has been previously demonstrated to exist on the $\mathrm{Au}(111)$ surface, following sulfur deposition and annealing to $450 \mathrm{~K}$. We introduce theoretical techniques within density functional theory to take into account charge transfer in an incommensurate system and model scanning tunneling microscopy images, which are in good agreement with experiment. Our simulations indicate that this model is remarkably robust. We analyze the nature of bonding in this structure using state-of-the-art Wannierfunction based techniques. Our analysis provides a natural explanation for the extraordinary robustness and unusual stoichometry of this layer. This structure and its chemistry have implications for related S-Au interfaces, such as those in self-assembled monolayers of thiols on Au substrates.
\end{abstract}




\section{Main text}

\section{Introduction}

Nano-structured materials, such as two-dimensional confined systems, have attracted immense interest because their structural and electronic properties often differ from those of bulk materials. ${ }^{1,2}$ These systems are promising candidates for many technological applications, including molecular electronic devices, sensors and catalysts. $^{2-4}$ Advances in nano-scale growth methods have produced a wealth of systems with interesting properties, ${ }^{3-5}$ but progress is often hampered by the lack of an atomisticscale understanding of their structure, which can be rather complex. In particular, incommensurate structures, which are not uncommon, defy theoretical analysis because the layer and substrate cannot both be treated exactly within a common unit cell.

In this work, we revisit the structure of an incommensurate nano-scale system which is particularly intriguing: a two-dimensional (2D) ordered layer of gold sulfide, formed on the $\mathrm{Au}(111)$ surface following sulfur deposition and annealing at $450 \mathrm{~K}^{5,6}$ What is interesting about this layer is that it provides fresh insights into the nature of possible precursor states for the bonding of organic molecules (such as alkylthiols) to $\mathrm{Au}$ via sulfur, systems that are of great interest in technological applications. ${ }^{3}$ Both the incommensurate nature and the unusual stoichiometry of this layer required the development of new theoretical tools in the framework of first-principles calculations. These tools provide a comprehensive picture of the structure and chemistry of the sulfide monolayer that has important implications for a wider range of related applications.

\section{Methods}


The scanning tunneling microscopy (STM) experiments were performed in ultrahigh vacuum with a base pressure of $4 \times 10^{-10}$ Torr. The Au(111) surface was cleaned by $\mathrm{Ar}^{+}$sputtering at $300 \mathrm{~K}$, followed by annealing to $700 \mathrm{~K}$ for $10 \mathrm{~min}$ and $600 \mathrm{~K}$ for 60 min. The characteristic herringbone reconstruction was observed following this procedure. $\mathrm{SO}_{2}$ ("Matheson", anhydrous grade) was introduced by chamber backfilling. Only a small fraction of the $\mathrm{SO}_{2}$ decomposes and deposits sulfur on the $\mathrm{Au}(111)$ surface, as monitored by Auger electron spectroscopy. Importantly, no oxygen-containing species was detected on the surface at any time, suggesting that the oxygen released during $\mathrm{SO}_{2}$ decomposition is removed by an abstraction reaction with excess $\mathrm{SO}_{2}{ }^{7}$ Further experimental details can be found in Reference 5 .

All our calculations were performed in the framework of density functional theory with the generalized gradient approximation for the exchange-correlation functional $(\mathrm{PW}-91)^{8}$. A plane-wave basis set was used, with scalar relativistic pseudopotentials to represent the atomic cores. $4 \times 4$ and $8 \times 8 k$-point meshes were used for calculations with and without the Au substrate respectively. Typically, the total energy for structures we considered is well-converged with a $3 \times 3 k$-point mesh. At least $10 \AA$ of vacuum was used in each calculation to separate the slab geometries, and convergence of relevant physical quantities was checked with respect to vacuum size. Within this framework, we introduced new theoretical approaches to obtain first, the atomic structure, and second, the bonding characteristics, of the incommensurate $\mathrm{AuS}$ layer on $\mathrm{Au}(111)$.

In calculations for the atomic structure, we used the projected augmented wave method $^{9}$ with an energy cutoff of $280 \mathrm{eV}$, as implemented in VASP. The Au substrate was represented by a slab of $6 \mathrm{Au}(111)$ layers, the bottom 3 of which are frozen in their 
bulk positions. Geometry optimization was performed with a force convergence criterion of $0.05 \mathrm{eV} / \AA$. The resulting structures were used for analysis of bonding characteristics, by constructing localized Wannier functions from the Kohn-Sham wavefunctions.

Further details of our theoretical approaches will be described below.

\section{Experimental results}

We have previously reported detailed scanning probe studies of the interaction of sulfur with $\mathrm{Au}(111) .{ }^{5,6}$ These studies established that sulfur interacts with $\mathrm{Au}(111)$ in a dynamic, rather than static, manner, with large scale mass transport and the dislodgement of Au terrace atoms to form a gold-sulfide phase.

In short, STM images ${ }^{5}$ show that a sulfur coverage of as low as $0.1 \mathrm{ML}$ completely lifts the herringbone surface reconstruction of $\mathrm{Au}(111)$ even at room temperature $(300 \mathrm{~K})$. At $0.3 \mathrm{ML}$, an ordered $(\sqrt{\mathbf{3}} \times \sqrt{\mathbf{3}}) \mathrm{R} 30^{\circ}$ adlayer of adsorbed sulfur atoms is formed. Above this coverage, a dynamic rearrangement of the Au surface occurs, with small islands and monatomic etch pits nucleating on Au terraces, strongly suggesting that $\mathrm{Au}$ atoms are removed from terraces into a growing gold sulfide phase that is distinct from that of adsorbed sulfur observed at lower coverages. Similar incorporation of stoichiometric amounts of substrate atoms into adsorbate-induced surface adlayers has been observed in other systems, such as a 2D oxide layer on $\operatorname{Pd}(111)^{10}$ and a $2 \mathrm{D}$ sulfide on $\mathrm{Al}(111)^{11}$. At a saturation coverage of $0.6 \mathrm{ML}$, the surface takes on a sponge-like morphology that is completely covered by a 2D layer. Quasirectangular ring-like structures with some short-range order are formed when the system is subsequently annealed to $420 \mathrm{~K}$. Similar features have been observed during the electrochemical deposition of $\mathrm{S}$ on $\mathrm{Au} ;{ }^{12}$ it was proposed that these rings correspond to 
strained $\mathrm{S}_{8}$ molecules. Further annealing to $450 \mathrm{~K}$ leads to Ostwald ripening of the original etch pits, resulting in large vacancy islands of monatomic depth. The S coverage drops to approximately $0.5 \mathrm{ML}$ and a 2D layer with long-range order completely covers the Au surface. High-resolution STM images ${ }^{5}$ of this ordered 2D phase reveal that the system is incommensurate, with a $(8.8 \pm 0.4) \times(8.2 \pm 0.4) \AA^{2}$ unit cell and an angle of $82^{\circ}$ $\pm 4^{\circ}$ between the lattice vectors. Based on the areas of the vacancy islands, it was estimated that approximately $0.5 \mathrm{ML}$ of Au is incorporated into the ordered 2D sulfide layer, suggesting a 1:1 Au-S stoichiometry. ${ }^{5}$ This stoichiometry is distinct from those of bulk gold sulfides, $\mathrm{Au}_{2} \mathrm{~S}$ and $\mathrm{Au}_{2} \mathrm{~S}_{3}{ }^{13}$

\section{Atomic structure: Approach and model}

The unusual stoichiometry and 2D nature of the gold sulfide layer suggest that it is a novel phase distinct from 3D bulk gold sulfides. To understand this interesting phase, we proceed to construct an atomic-scale model for this system. The incommensurate nature implies that the AuS layer does not interact strongly with the Au surface (this picture is later confirmed using Wannier orbital analysis of bonding). We therefore consider the system in two stages. First, we determine the atomic structure of an isolated AuS layer in a fixed unit cell consistent with experimental measurements, and in a fully relaxed cell. Next, we analyze how the substrate affects the atomic and electronic structure of this layer, the latter in a manner which takes into account the incommensurate nature of the interaction by averaging over several different configurations.

In the first step, given the $\mathrm{Au}(111)$ surface lattice, a natural choice for the fixed unit cell is given by the black box in Fig. 1(a): this cell has a lattice constant of $8.65 \AA$ (3 
times that of $\mathrm{Au}$ ) in direction $\boldsymbol{a}_{1}$, and a lattice angle of $79^{\circ}$, both values within experimental error bars $(8.65 \AA$ is within the range of $8.4 \AA$ to $9.2 \AA$ for the first lattice constant). The lattice constant in direction $\boldsymbol{a}_{2}$ was fixed at the experimental value of 8.20 $\AA$, which is not a simple multiple of the Au lattice constant. We considered several models with different numbers of atoms per unit cell, with stoichiometry $\mathrm{Au}: \mathrm{S}=1: 1$, and different arrangements of these atoms, using for guidance information on the local coordination chemistry of $\mathrm{Au}$ and $\mathrm{S}$ in known compounds. ${ }^{14,15}$ Fully relaxing the positions of these atoms within the fixed unit cell resulted in only one stable structure (in all other structures, the atoms rearranged drastically and the atomic forces sometimes did not converge). The stable structure, which we call $A$, is planar with $4 \mathrm{Au}$ and $4 \mathrm{~S}$ atoms per unit cell (Fig. 1(b)). Details of bond lengths are given in Table 1(a). The corresponding S coverage is $0.41 \mathrm{ML}$, assuming that a completely flat, unreconstructed $\mathrm{Au}(111)$ surface is entirely covered by the AuS layer. This coverage is close to the experimental estimate of $0.5 \mathrm{ML}$, taking into account the attachment of $\mathrm{S}$ atoms to the edges of Au vacancy islands present on the annealed, sulfide-covered $\mathrm{Au}(111)$ surface, and possible uncertainty in the experimental calibration.

We next allowed the unit cell parameters to relax without any constraints: this resulted in an almost uniform shrinking of the unit cell vectors. The lattice angle changed to $78^{\circ}$ (which is within experimental error bars and close to the corresponding angle in $A$ of $79^{\circ}$ ). The new lattice constants are $7.85 \AA$ and $7.65 \AA$, which are respectively $9.3 \%$ and $6.7 \%$ smaller than the corresponding lattice constants in $A$. However, the ratio between lattice constants is 1.04 , close to the corresponding ratio of 1.05 in $A$. The atomic geometry also remains very similar to that in $A$ (Fig. 2(a)). This shows that the 
atomic structure and unit cell shape in $A$ are robust. The relaxed unit cell is too small compared to experimental values (the lower bound in experiment being $8.4 \times 7.8 \AA^{2}$ ). However, the shrinking of the unit cell is consistent with the shorter bond lengths found in bulk $\mathrm{Au}_{2} \mathrm{~S}^{14}$ and other compounds with Au-S bonds ${ }^{15}$ (Table 1). As discussed below, the larger unit cell observed in experiment is stabilized by charge transfer from the Au substrate.

We now consider the effects of the Au substrate. First, we examine this effect on important structural features of the AuS monolayer. Although the combined system is incommensurate, it is possible to fit the AuS unit cell in a supercell of the $\mathrm{Au}(111)$ surface by using the equilibrium Au lattice constant predicted from calculations on bulk $\mathrm{Au}\left(c_{\text {theory }}=2.948 \AA\right)$ instead of the experimental value $\left(c_{\text {expt }}=2.884 \AA\right)$. In this arrangement, the gray area in Fig. 1(a), which is commensurate with the Au lattice, has dimensions of $(8.84 \times 7.80) \AA^{2}$, which are still within experimental error bars for the AuS unit cell ( $7.80 \AA$ is within the range of $7.8 \AA$ to $8.6 \AA$ for the second lattice constant). We can now perform geometry optimizations for a periodic system with a supercell containing a AuS layer on top of a 6-layered $\mathrm{Au}(111)$ slab. In the most stable structure, which we call $B$, the 2-fold coordinated $\mathrm{Au}$ atom $\left(\mathrm{Au}_{(2)}\right)$ in $\mathrm{AuS}$ is at site $\mathbf{X}$ and the 4 -fold coordinated $\mathrm{Au}$ atom $\left(\mathrm{Au}_{(4)}\right)$ at site $\mathbf{O}$ (Fig. 1). Initial structures with $\mathrm{Au}_{(2)}$ positioned at any of the three-fold sites of the surface layer, also relaxed to structure $B$. If $\mathrm{Au}_{(4)}$ is placed initially at $\mathbf{X}$ and $\mathrm{Au}_{(2)}$ at $\mathbf{O}$, each of these $\mathrm{Au}$ atoms remains at its initial site during geometry optimization. However, the remaining atoms completely rearrange to eventually yield the same structure (B), with the Au atom at site $\mathbf{X}$ becoming 2-fold 
coordinated, and that at $\mathbf{O}$ becoming 4 -fold coordinated. This indicates that the AuS structure is favored and remarkably robust even in the presence of the Au substrate.

The incommensurate arrangement and long-range order of the AuS layer imply that the layer should feel an average effect of the substrate. This average effect is not altered as the relative position of the overlayer is varied. The calculations mentioned above are not useful in describing this effect because the forced matching of lattice constants between the overlayer and substrate introduces artificial corrugations for some atomic positions. Therefore, in analyzing the electronic features of the AuS layer, it is necessary to introduce a different approach to take into account the average effects of the substrate in the incommensurate system.

As the overlayer and substrate interact weakly with each other, and are both metallic, charge transfer is expected to be the dominant electronic effect of the substrate. The weak interaction with the substrate also suggests that the AuS atomic geometry and band structure will remain largely unchanged by the Au substrate, allowing us to use the structural features of the stable isolated layer A, and model charge transfer by changing the occupancy of the AuS bands (so-called 'rigid band model'). We model the change in occupancy by shifting the Fermi level $\left(\mathrm{E}_{\mathrm{F}}\right)$ of $A$ by an amount $\Delta \mathrm{E}_{\mathrm{F}}$ to that expected for the combined system.

To estimate $\Delta \mathrm{E}_{\mathrm{F}}$, we first construct a series of models that are representative of the different relative positions that the incommensurate AuS layer can take on the $\mathrm{Au}(111)$ substrate. Next, we compute the average of the substrate-induced shifts in Fermi level at each of these positions. The models were constructed as follows. First, we relax atoms in the top $3 \mathrm{Au}(111)$ layers of the 6-layer $\mathrm{Au}(111)$ slab, and atoms in the $\mathrm{AuS}$ 
layer, with the additional constraints that the $\mathrm{AuS}$ layer be planar, with $\mathrm{Au}_{(2)}$ at $\mathbf{X}$ and $\mathrm{Au}_{(4)}$ at $\mathbf{O}$. The optimal height of the AuS layer above the Au surface is $2.53 \AA$, which is the same as the average height of the layer above the Au surface is in structure $B$. We call the resulting system $B^{\prime}$. Next, we shift this AuS layer in steps by $\lambda \boldsymbol{a}_{2}$ relative to the substrate $(\lambda=0.0,0.1, \ldots, 0.9)$. At each step, only the top $3 \mathrm{Au}(111)$ layers are allowed to relax, resulting in systems which we call $B_{\lambda}$.

We calculate the substrate-induced shift in Fermi level $\Delta \mathrm{E}_{\mathrm{F}}$ by taking the average over $\lambda$ of the differences in work functions $\Delta \Phi$ between the metallic systems $A$ and $B_{\lambda}$ '. The work function of the systems are calculated using symmetric slabs, obtained by taking mirror images about the 3 frozen $\mathrm{Au}(111)$ layers, to give 9-layered Au slabs covered on both sides by AuS. The work function $\Phi$ is computed as $V_{\text {vac }}{ }^{(c)}-E_{F}{ }^{(c)}$, where $\mathrm{E}_{\mathrm{F}}{ }^{(\mathrm{c})}$ and $\mathrm{V}_{\text {vac }}{ }^{(\mathrm{c})}$ are the Fermi level and vacuum potential in the calculation. As described below, this approach allows us to estimate the effects of charge transfer remarkably well.

\section{Atomic structure: Results}

The estimated $\mathrm{E}_{\mathrm{F}}$ of the incommensurate $\mathrm{AuS}$ layer on $\mathrm{Au}(111)$ is found to be $(0.85 \pm 0.03) \mathrm{eV}$ closer to the vacuum potential than $\mathrm{E}_{\mathrm{F}}$ of $A$, corresponding to electronic charge transfer from $\operatorname{Au}(111)$ to $A$. This is consistent with the larger work function of $A$ $(6.18 \mathrm{eV})$ relative to $\mathrm{Au}(111)$, which we calculate to be $5.18 \mathrm{eV}$, in reasonable agreement with the experimental value ${ }^{16}$ of $5.31 \mathrm{eV}$.

We can now simulate STM images using $A$ with $\Delta \mathrm{E}_{\mathrm{F}}=0.85 \mathrm{eV}$, within the Tersoff-Hamann approximation. ${ }^{17}$ To take into account the effect of convolution between sample and tip wave functions, ${ }^{17}$ as well as the small amount of spot broadening in the scan direction $\boldsymbol{x}$, we use elliptical Gaussian broadening with standard deviations $s_{x}$ of 1.2 
$\AA$ and $s_{y}$ of $0.8 \AA$ (both less than half of the bond lengths in the structure). Our simulations reproduce convincingly the high-magnification experimental images at 2 different sample bias voltages (Fig. 3), and are insensitive to the exact values of $s_{x}$ and $s_{y}$. The calculations indicate that the bright spots in the STM images in Fig. 3 are associated with positions of the $\mathrm{S}_{(3, \mathrm{a})}$ and $\mathrm{S}_{(3, \mathrm{~b})}$ atoms.

\section{Nature of bonding: Approach}

The fact that $\mathrm{Au}$ atoms from terraces can be incorporated into a stable, incommensurate AuS layer is quite remarkable. This warrants a closer examination of bonding in the AuS layer. In Fig. 4, the charge density difference between structure $A$ (Fig. 1(b)) and the superposition of atomic densities is plotted in the plane of the structure. From this plot, it is evident that charge accumulates between the Au and $\mathrm{S}$ atoms, seemingly closer to the S. A small amount of charge accumulation seems to be present between $\mathrm{Au}_{(3, \mathrm{a})}$ and $\mathrm{Au}_{(3, \mathrm{~b})}$ as well. In order to obtain more detailed chemical insights than that provided by Fig. 4 , we use a recently developed scheme ${ }^{18}$ which provides an excellent description of bonding in well-characterized systems of both metallic and covalent nature. The analysis relies on the successive construction of two sets of localized Wannier orbitals with initially-specified centers and symmetries (e.g. atomic $s, p$ or $d$ symmetries). The first set (I) consists of atom-centered orbitals (AOs), and the second (II) of both AOs and bond-centered orbitals (BOs).

The construction of Wannier orbitals suited for the current application is described in detail in Reference 18. Briefly, we make a choice of symmetry properties of Wannier functions specified with (a) the centre of the Wannier function and (b) the irreducible representation of its site symmetry group given in terms of its partner 
function, for example a spherical harmonic. Such a choice is typically guided by the symmetry properties of Bloch functions at high symmetry points in the Brillouin zone, and is self-corrective, as discussed below. Well-localized Wannier functions can be obtained if they are Fourier transformed from Bloch functions that are smooth and periodic in Bloch vector $\mathbf{k}$. As described in Reference 18, these Bloch functions can in turn be obtained by introducing an auxiliary subspace. This auxiliary subspace is constructed from highly localized functions of the chosen symmetry (spherical harmonic for the angular part, and a Gaussian form for the radial part). These highly localized and orthonormal orbitals are Fourier transformed to obtain Bloch functions that span the auxiliary subspace. The key point is that these Bloch functions have the same symmetry properties as those of the Bloch functions in the physical subspace of occupied (and some of the unoccupied in metals) electronic states in the system. A unitary transformation is performed on the Bloch functions in the physical subspace such that the overlap matrix between Bloch states of the auxiliary and the physical subspace becomes hermitian, amounting to vanishing of open path non-abelian geometric phases. This gives the desired Bloch functions and corresponding well-localized Wannier functions.

Determination of the unitary transformation is facilitated by singular value decomposition of the overlap matrix. The scheme is self-corrective in the sense that some of the singular values vanish in the case where the choice of symmetry of the auxiliary subspace is not quite optimal and hints for correction.

Another important feature of our approach ${ }^{18}$ is that Bloch eigenfunctions are weighted by the square-root of their occupation numbers in the above-described transformation. This allows treatment of metallic systems considered here. Further, the 
resulting Wannier functions are no longer constrained to have unit charge as usual, but have an integrated charge that reflects the physics of the system. For example, the amount of charge in each localized $\mathrm{AO}$ and $\mathrm{BO}$ is directly related to atomic oxidation states and relative bond strengths respectively.

\section{Nature of bonding: Results}

We use here two choices of auxiliary subspaces: (I) one with only atom-centered orbitals (AOs), and (II) one with AOs and bond-centered orbitals (BOs), by including more unoccupied states in the physical subspace. We first illustrate the method by discussing results for the stable structure $A$ (Fig. 1(b)). The AOs for Au 6s (Fig. 5(a-c)), S $3 s$ (Fig. 5(d)) and S 3p (Fig. 5(e-f)) electrons are spatially extended, indicating that these electrons contribute substantially to bond covalency. In particular, the singular value for a Au-centered AO with $s$-symmetry vanishes, reflected in the distortion of these AOs from atomic-like $s$ orbitals (Fig. 5(a-c)), in contrast to the S AOs that still resemble atomic-like $s$ and $p$ orbitals (Fig. 5(e-f)). This suggests an especially important role of Au $6 s$ electrons in the covalency of bonds in the AuS layer. On the other hand, the Au $6 p$ AOs are unoccupied and are especially localized, suggesting that the $6 p$ orbitals of Au do not hybridize with $6 s$ orbitals during bond formation. Au $5 d$ AOs, although not spatially extended, are less localized than the $6 p$ AOs, and as we discuss later, do contribute to bond formation. This is consistent with the general argument in the literature that electronegative ligands of $\mathrm{Au}$ support $\mathrm{Au} 5 d$ participation in bond formation, while electropositive ligands support $\mathrm{Au} 6 p$ participation: ${ }^{19}$ the electronegativity of $\mathrm{S}(\mathrm{Au})$ is 2.58 (2.54). The lack of $6 s-6 p$ hybridization is also consistent with the relatively large energy separation between $6 s$ and $6 p$ levels compared to $6 s$ and $5 d$ levels in atomic Au. ${ }^{20}$ 
The amount of electron charge in $\mathrm{Au} \mathrm{AOs}$ is largest for $\mathrm{Au}_{(2)}$ and smallest for $\mathrm{Au}_{(4)}$, consistent with formal oxidation states expected from the literature: $\mathrm{Au}^{\mathrm{III}}$ and $\mathrm{Au}{ }^{\mathrm{I}}$ have square planar and linear coordination geometries respectively, while the $5 d^{9}$ configuration in $\mathrm{Au}^{\mathrm{II}}$ is typically accompanied by a $\mathrm{Au}-\mathrm{Au}$ bond. ${ }^{20}$ Indeed, the ring, $\mathrm{Au}_{(4)^{-}}$ $\mathrm{S}_{(3, \mathrm{~b})}-\mathrm{Au}_{(3, \mathrm{~b})}-\mathrm{S}_{(2, \mathrm{~b})}-\mathrm{Au}_{(4)}-\mathrm{S}_{(3, \mathrm{a})}-\mathrm{Au}_{(3, \mathrm{a})}-\mathrm{S}_{(2, \mathrm{a})}$, is a motif found in $\mathrm{Au}^{2+}$ compounds. ${ }^{15,20} \mathrm{~S}$ is known to form bonds with Au in all three oxidation states; ${ }^{15}$ the 2 - and 3-fold coordination for $\mathrm{S}$ is similar to that for $\mathrm{O}$ in $\mathrm{Au}_{2} \mathrm{O}_{3}{ }^{21}$ (the structure of $\mathrm{Au}_{2} \mathrm{~S}_{3}$ is unknown). The 1:1 stoichiometry in structure $A$ thus arises from having one $\mathrm{Au}^{\mathrm{III}}\left(\mathrm{Au}_{(4)}\right)$, one $\operatorname{Au}^{\mathrm{I}}\left(\mathrm{Au}_{(2)}\right)$ and two $\mathrm{Au}^{\mathrm{II}}\left(\mathrm{Au}_{(3, \mathrm{a})}\right.$ and $\left.\mathrm{Au}_{(3, \mathrm{~b})}\right)$ atoms per unit cell, in contrast to bulk gold sulfides $\left(\mathrm{Au}_{2} \mathrm{~S} \text { and } \mathrm{Au}_{2} \mathrm{~S}_{3}\right)^{13}$ which contain purely $\mathrm{Au}^{\mathrm{I}}$ or $\mathrm{Au}{ }^{\mathrm{III}}$ respectively.

Each $\mathrm{Au}(\mathrm{S})$ atom contributes $0.3-0.4 e(0.8-0.9 e)$ per bond. The Au-S bonds are partially polar, as indicated by their asymmetric BOs (Fig. 6). This is consistent with excess charge in S $3 p$ AOs and shortage of charge in Au $6 s$ (and some 5d) AOs. The origin of $\mathrm{Au}-\mathrm{Au}$ interactions in $\mathrm{Au}{ }^{\mathrm{II}}-\mathrm{Au}^{\mathrm{II}}$ (and $\mathrm{Au}-\mathrm{A} u^{\mathrm{I}}$ ) compounds has been the subject of considerable debate. ${ }^{19}$ Previous ab initio studies have described the $\mathrm{Au}^{\mathrm{II}}-\mathrm{Au}^{\mathrm{II}}$ interaction as a single covalent bond with considerable $6 s-6 s$ character. ${ }^{19}$ Our calculations indicate that the $6 \mathrm{~s}$ electrons indeed are key players in the $\mathrm{Au}-\mathrm{Au}$ bond in the isolated $\mathrm{AuS}$ layer. We further predict that the $\mathrm{Au}-\mathrm{Au}$ bond in isolated $\mathrm{AuS}$ is stabilized by delocalization over $S_{(2, a)}$ and $S_{(2, b)}$, as indicated by the multi-centered $\mathrm{Au}_{(3, \mathrm{a})}-\mathrm{Au}_{(3, \mathrm{~b})} \mathrm{BO}$ in Fig. 6(c). In addition, the amount of charge in the $\mathrm{Au}_{(3, \mathrm{a})}-\mathrm{Au}_{(3, \mathrm{~b})} \mathrm{BO}$ is $20-27 \%$ less than that in the Au-S BOs, indicating that the Au-Au bond strength in isolated AuS is weaker than other bonds in the layer. 
To understand the effects of the substrate, we performed a similar analysis on structure $B\left(\mathrm{Au}_{(2)}\right.$ at site $\mathbf{X}$ on $\mathrm{Au}(111)$ and $\mathrm{Au}_{(4)}$ at $\mathbf{O}$ (Fig. 1)), with $\mathrm{AOs}$ centered on atoms in the AuS layer and the top $\mathrm{Au}(111)$ layer. The shapes of Au-S BOs are not affected by the substrate (Fig. 7(a-b)), while the multi-centered Au-Au BO, though slightly extended towards the substrate atoms, remains largely confined within the AuS plane (Fig. 7(c-d)). The fact that BOs within the AuS layer largely retain their shapes even in the presence of the $\mathrm{Au}(111)$ layers suggests that the AuS layer does not interact strongly with the substrate, which is consistent with the experimentally observed incommensurability and our theoretical assumptions.

Compared to the isolated layer, the electronic charge in each $\mathrm{BO}$ increases by 54 $\%$ on average, except for the $\mathrm{Au}-\mathrm{Au} \mathrm{BO}$, where the increase is $15 \%$. In contrast, the contribution of each AO to bonding either decreases, or increases by at most $9 \%$. This implies that bonds within the AuS layer are strengthened at the expense of substrate electrons, which may explain the robustness of the AuS structure. Using $\Delta \mathrm{E}_{\mathrm{F}}$ of $0.85 \mathrm{eV}$ and the DOS of the isolated layer $A$, we estimate the quantity of charge transferred to the layer to be $\sim 3.3 e$ per unit cell of $A$. Completely relaxing the isolated AuS layer in the presence of this extra charge does not change the atomic arrangements significantly (Fig. 2(b)). Importantly, however, the optimized lattice constants of the charged layer are $8.4 \AA$ and $7.9 \AA$ : these are near the low end but within the respective experimental ranges of [8.4 - 9.2] $\AA$ and [7.8 - 8.6] $\AA$ (see Table 1(a) for bond lengths). The lattice angle of $76^{\circ}$ is also reasonably close to the experimental range of $\left[78^{\circ}-86^{\circ}\right]$. These results are in contrast to what we found for the neutral AuS layer, where the optimized lattice constants were too small compared to experiment. This further confirms that charge 
transfer from the substrate stabilizes the stretched bonds in the supported layer, and that our estimated shift in $\mathrm{E}_{\mathrm{F}}$ models the effect of charge transfer reasonably well.

\section{Concluding Remarks}

As previously established in detailed scanning probe studies, ${ }^{5,6}$ the $\mathrm{Au}(111)$ surface interacts with deposited sulfur in a dynamic, rather than static, manner, eventually resulting in a $2 \mathrm{D}$ incommensurate $\mathrm{Au}-\mathrm{S}$ phase upon annealing to $450 \mathrm{~K}$. STM alone was insufficient to decipher the atomic structure of the Au-S phase, since STM images the electronic, rather than atomic, structure. In this work, we revisit the structure of this intriguing incommensurate phase, and discuss in detail an atomic-scale model for the system, which reproduces convincingly high-magnification STM images. What is striking about this model is that it is remarkably robust, even in the presence of the $\mathrm{Au}$ substrate. Furthermore, the structure reflects the rich co-ordination chemistry of Au, which is also present in Au compounds synthesized from Au ions in solution or gas phase. ${ }^{19,20}$ We provide a natural explanation for the remarkable robustness of the model, in terms of charge transfer from the substrate, bond types and formal oxidation states of $\mathrm{Au}$.

While we have not proven that the proposed model is the thermodynamically stable structure, the extraordinary robustness of the model and our bonding analysis indicate that it is strongly favored, and would therefore at least be an important precursor state. Together with STM studies, it is clear that the Au(111) surface is not simply an 'inert' surface, but can interact dynamically with deposited sulfur, with the incorporation of $\mathrm{Au}$ atoms from terrace sites into a sulfide adlayer at higher coverages. Our results suggest that the ring-like features reported in the literature ${ }^{12}$ may not simply be $\mathrm{S}_{8}$ 
molecules. Similar adsorbate-induced mobilization of Au atoms has been observed when oxygen atoms are deposited onto $\mathrm{Au}(111)$, resulting in a gold oxide adlayer. ${ }^{6}$

The dynamic nature of the $\mathrm{Au}(111)$ surface and the incorporation of $\mathrm{Au}$ terrace atoms into a sulfide adlayer in this system have important implications for the structure of the S-Au interface in self-assembled monolayers (SAMs) of thiols on $\mathrm{Au}(111),{ }^{3}$ which is crucial to determining their transport properties. ${ }^{22}$ Similar etch pits and islands have been observed in these systems, suggesting that Au terrace atoms will have similar interaction chemistry with thiol chains. The interface structure in thiol/Au systems, however, has commonly been interpreted in terms of a flat $\mathrm{Au}(111)$ surface, ${ }^{3}$ with only a few works ${ }^{23}$ proposing an interface structure involving Au vacancies. The latter involve ab initio calculations which indicate that the adsorption of methylthiolate on $\mathrm{Au}(111)$ is stabilized by the introduction of vacancies in the Au substrate, with the increased adsorption energy more than compensating for the vacancy formation energy. ${ }^{23}$ Our observations are consistent with this picture and further highlight the importance of considering composite Au-thiol adlayer systems in contrast to conventionally assumed adsorption on flat $\mathrm{Au}(111)$.

Furthermore, we have introduced theoretical techniques to take into account charge transfer and thereby simulate STM images for an incommensurate system without requiring exceedingly large supercells. We suggest that in general, the shift in Fermi level can be obtained by comparing an experimentally determined work function of the combined incommensurate system with a quantitative theoretical estimate of the work function of the isolated adlayer. This would render possible computationally tractable first-principles studies of many incommensurate systems involving metallic adlayers on 
metal substrates. We have also employed state-of-the-art Wannier function-based methods to yield detailed chemical insights into the nature of bonding in the system, and this analysis is applicable to a wide range of complex systems. 


\section{References}

1 G. Kresse, M. Schmid, E. Napetschnig, M. Shishkin, L. Kohler, and P. Varga, Science 308 (5727), 1440 (2005).

2 C. H. Ahn, K. M. Rabe, and J. M. Triscone, Science 303 (5657), 488 (2004).

3 J. C. Love, L. A. Estroff, J. K. Kriebel, R. G. Nuzzo, and G. M. Whitesides, Chem. Rev. 105 (4), 1103 (2005).

C. T. Campbell, Surf. Sci. Rep. 27 (1-3), 1 (1997).

M. M. Biener, J. Biener, and C. M. Friend, Langmuir 21 (5), 1668 (2005).

B. K. Min, A. R. Alemozafar, M. M. Biener, J. Biener, and C. M. Friend, Topics in Catalysis 36 (1-4), 77 (2005).

$7 \quad$ M. M. Biener, J. Biener, and C. M. Friend, Surf. Sci. 590 (2-3), L259 (2005).

J. P. Perdew and Y. Wang, Phys. Rev. B 45 (23), 13244 (1992).

$9 \quad$ P. E. Blochl, Phys. Rev. B 50 (24), 17953 (1994).

10 E. Lundgren, G. Kresse, C. Klein, M. Borg, J. N. Andersen, M. De Santis, Y. Gauthier, C. Konvicka, M. Schmid, and P. Varga, Phys. Rev. Lett. 88 (24), 246103 (2002).

11 T. Wiederholt, H. Brune, J. Wintterlin, R. J. Behm, and G. Ertl, Surf. Sci. 324 (23), 91 (1995).

12 X. P. Gao, Y. Zhang, and M. J. Weaver, J. Phys. Chem. 96 (11), 4156 (1992); C. Vericat, G. Andreasen, M. E. Vela, and R. C. Salvarezza, J. Phys. Chem. B 104 (2), 302 (2000); C. Vericat, M. E. Vela, G. Andreasen, R. C. Salvarezza, L. Vazquez, and J. A. Martin-Gago, Langmuir 17 (16), 4919 (2001); C. Vericat, M. E. Vela, G. A. Andreasen, R. C. Salvarezza, F. Borgatti, R. Felici, T. L. Lee, F. 
Renner, J. Zegenhagen, and J. A. Martin-Gago, Phys. Rev. Lett. 90 (7), 075506 (2003).

J. C. Bailar, H. J. Emeleus, R. Nyholm, and A. F. Trotman-Dickenson, Comprehensive inorganic chemistry. (Pergamon Press, 1973). (1995). H. Schmidbaur, Gold: progress in chemistry, biochemistry and technology. (John Wiley \& Sons, 1999).

S. Hufner, Photoelectron Spectroscopy: Principles and Applications, 3 ed. (Springer-Verlag, Berlin Hiedelberg, 2003).

J. Tersoff and D. R. Hamann, Phys. Rev. Lett. 50 (25), 1998 (1983).

J. Bhattacharjee and U. V. Waghmare, Phys. Rev. B 73, 121102 (R) (2006).

$$
\text { P. Pyykko, Angew. Chem. Int. Edit. } 43 \text { (34), } 4412 \text { (2004). }
$$

R. J. Puddephatt, The Chemistry of Gold. (Elsevier, 1978).

P. G. Jones, H. Rumpel, E. Schwarzmann, G. M. Sheldrick, and H. Paulus, Acta Crystallogr. B 35 (JUN), 1435 (1979).

22 G. K. Ramachandran, T. J. Hopson, A. M. Rawlett, L. A. Nagahara, A. Primak, and S. M. Lindsay, Science 300 (5624), 1413 (2003); E. G. Emberly and G. Kirczenow, Phys. Rev. Lett. 91 (18), 188301 (2003); Z. Hens, D. V. Tallapin, H. Weller, and D. Vanmaekelbergh, Appl. Phys. Lett. 81 (22), 4245 (2002). C. C. Liew, and H. Nozoye, Surf. Sci. 514 (1-3), 389 (2002). 


\section{Acknowledgements}

We gratefully acknowledge support under the auspices of the Harvard NSEC which is funded by NSF Grant No. DMR-02-13805. SYQ thanks A-STAR, Singapore,

for fellowship support. J. Biener acknowledges current support under the auspices of the U.S. Department of Energy by the University of California, Lawrence Livermore National Laboratory. J. Bhattacharjee thanks CSIR, India, for a research fellowship. UVW acknowledges support from a DuPont Young Faculty Grant. This work was partially supported by NCSA under DMR040002. This work was performed under the auspices of the U.S. Department of Energy by University of California, Lawrence Livermore National Laboratory under Contract W-7405-Eng-48. 


\begin{tabular}{|c|c|c|c|c|}
\hline $\begin{array}{c}\text { Geometric } \\
\text { features }\end{array}$ & Structure $A$ & $\begin{array}{l}\text { Fully relaxed, } \\
\text { neutral layer }\end{array}$ & $\begin{array}{l}\text { Fully relaxed, } \\
\text { charged layer }\end{array}$ & Experiment \\
\hline$a_{1} \times a_{2}$ & $8.7 \times 8.2$ & $7.9 \times 7.7$ & $8.4 \times 7.9$ & $(8.8 \pm 0.4) \times(8.2 \pm 0.4)$ \\
\hline$\theta$ & $79^{\circ}$ & $78^{\circ}$ & $79^{\circ}$ & $82^{\circ} \pm 4^{\circ}$ \\
\hline $\begin{array}{l}A \mathbf{u}_{(2)}-\mathbf{S}_{(3)} \\
\left(\mathbf{A u} \mathbf{u}^{\mathrm{I}}-\mathbf{S}^{-I I}\right)\end{array}$ & 2.41 & 2.29 & 2.34 & $2.17^{14}$ \\
\hline $\begin{array}{c}A u_{(3)}-S_{(2)} \\
\left(A u^{I I}-S^{-I I}\right)\end{array}$ & 2.33 & 2.25 & 2.30 & - \\
\hline $\begin{array}{c}A u_{(3)}-S_{(3)} \\
\left(A u^{I I}-S^{-I I}\right)\end{array}$ & 2.41 & 2.28 & 2.35 & - \\
\hline $\begin{array}{c}A u_{(4)}-S_{(2)} \\
\left(A u^{I I I}-S^{-I I}\right)\end{array}$ & 2.45 & 2.37 & 2.42 & $2.30-2.35,2.40^{15}$ \\
\hline $\begin{array}{r}A u_{(4)}-S_{(3)} \\
\left(A u^{I I I}-S^{-I I I}\right)\end{array}$ & 2.58 & 2.37 & 2.43 & $2.30-2.35,2.40^{15}$ \\
\hline $\begin{array}{c}\mathbf{A} \mathbf{u}_{(3, \mathbf{a})}-\mathbf{A} \mathbf{u}_{(3, \mathrm{~b})} \\
\left(\mathbf{A} \mathbf{u}^{\mathrm{II}}-\mathbf{A} \mathbf{u}^{\mathrm{II}}\right)\end{array}$ & 2.87 & 2.88 & 2.85 & $2.60,3.10^{15}$ \\
\hline
\end{tabular}

Table 1. Geometric features in structure $A$, in the fully relaxed neutral and charged (3.3 e/cell) layers, and in experiment. $\left(a_{1} \times a_{2}\right)$ and $\theta$ denote respectively the lattice dimensions (in $\AA$ ) in the $\boldsymbol{a}_{\mathbf{1}}$ and $\boldsymbol{a}_{\mathbf{2}}$ directions, and the angle between lattice vectors for the AuS unit cell. The remaining rows tabulate bond lengths (in $\AA$ ) in the respective AuS models and in experiment. The experimental bond lengths are taken from the literature of known compounds that contain Au-S or Au-Au bonds with the same formal oxidation states as given in brackets in the first column. Specifically, the bond length for $\mathrm{Au}^{\mathrm{I}}-\mathrm{S}^{-\mathrm{II}}$ is taken from crystal data on bulk $\mathrm{Au}_{2} \mathrm{~S}^{14}{ }^{1}$ In compounds with $\mathrm{Au}^{\mathrm{III}}-\mathrm{S}^{-\mathrm{II}}$ bonds, typical $\mathrm{Au}^{\mathrm{III}}$ - 
$\mathrm{S}^{-\mathrm{II}}$ bond lengths are $2.40 \AA$ if $\mathrm{S}$ bridges two $\mathrm{Au}{ }^{\mathrm{III}}$ atoms, and 2.30-2.35 $\AA$ otherwise. ${ }^{15}$ The $A u^{I I}-A u^{I I}$ bond length is about $2.60 \AA$ for covalent $A u^{I I}-A u^{I I}$ bonds and $3.10 \AA$ for weaker $\mathrm{Au}{ }^{\mathrm{II}}-\mathrm{Au}{ }^{\mathrm{II}}$ aurophilic interactions. ${ }^{15}$ 

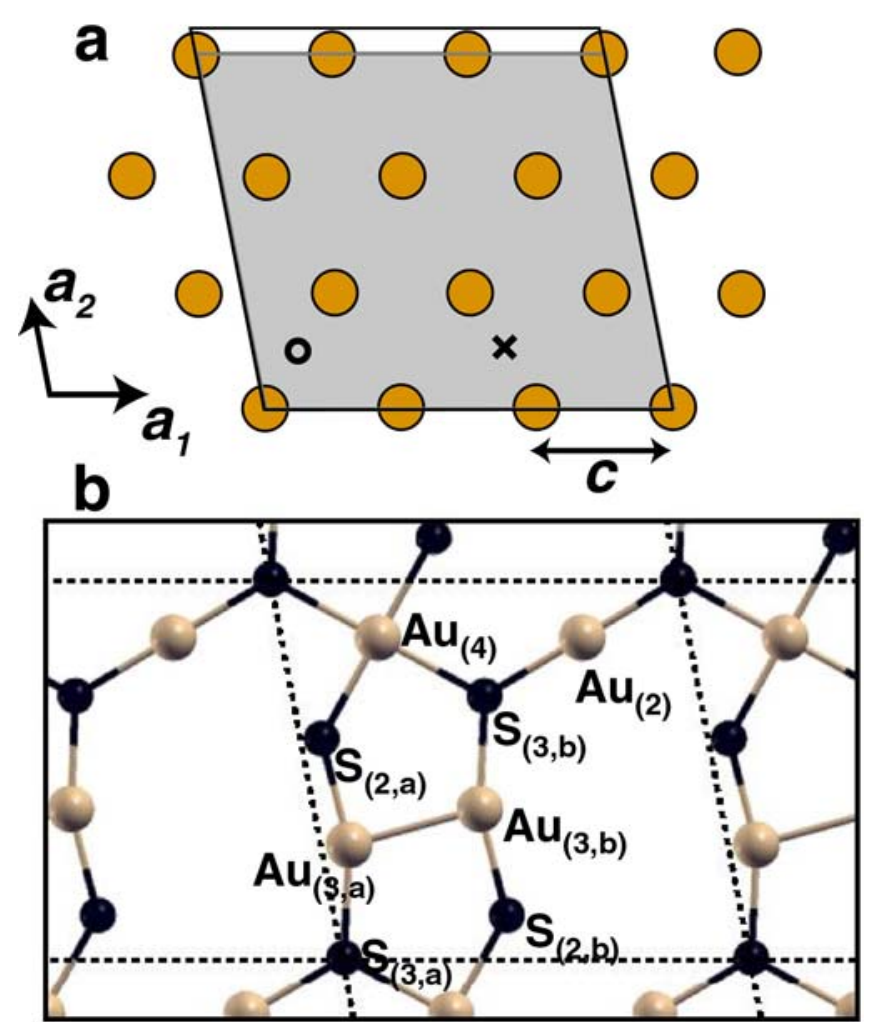

FIG. 1. (color online) (a) Proposed orientation of AuS unit cell (black box). (b)

Atomic structure of $\boldsymbol{A}$. Numerical subscripts denote the coordination of each atom and letter subscripts indicate inequivalent atoms of the same coordination. The cross and circle in (a) mark sites $\mathbf{X}$ and $\mathbf{O}$ respectively, relevant to structure $B$ described in the text. 

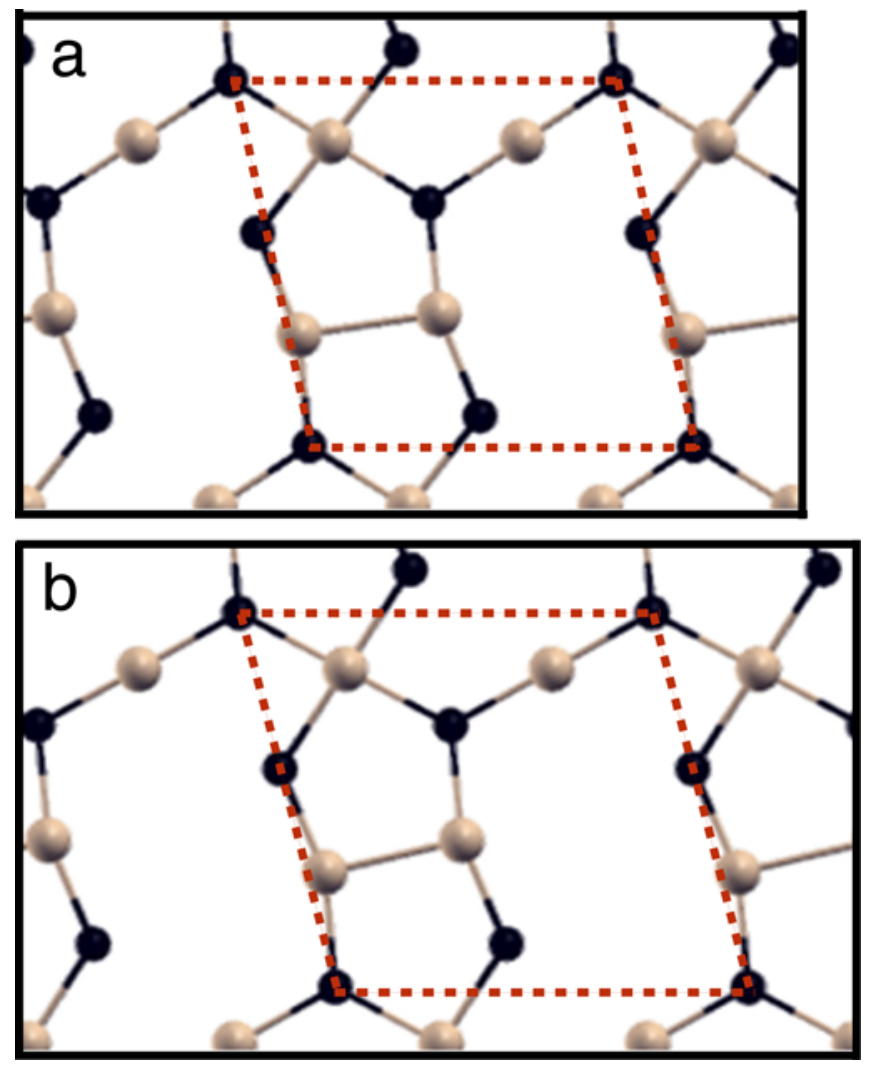

FIG. 2 (color online) Fully relaxed structures and unit cells (red boxes) for isolated,

(a) neutral and (b) charged AuS layers. The structure in (b) has a charge of $3.3 \mathrm{e} / \mathrm{cell}$. 


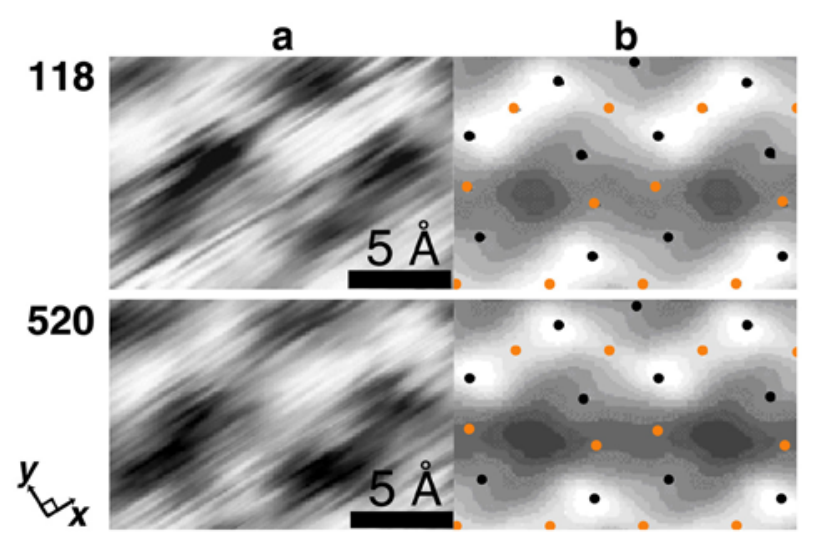

FIG. 3. (color online) (a) Constant height STM images collected at room

temperature, (b) STM simulations. Orange and black circles mark lateral positions of $\mathrm{Au}$ and $\mathrm{S}$ atoms respectively. The numbers indicate sample bias voltages in $\mathrm{mV}$, and the scan direction is given by $\boldsymbol{x}$. 


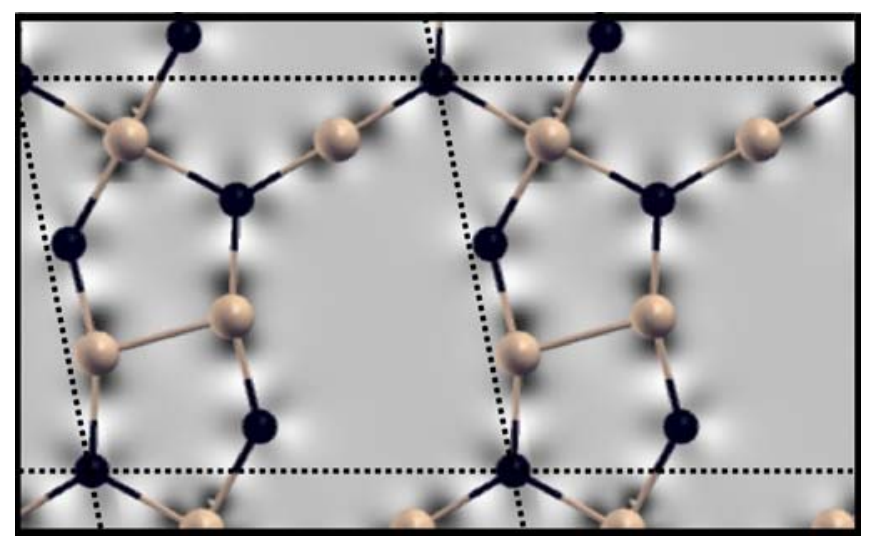

FIG. 4. (color online) Charge density difference plot. The charge density difference between structure $A$ and the superposition of atomic densities, is plotted in the plane of the structure; the scale runs from $-0.0005 \mathrm{e}$ (black) to $+0.0002 \mathrm{e}$ (white). 


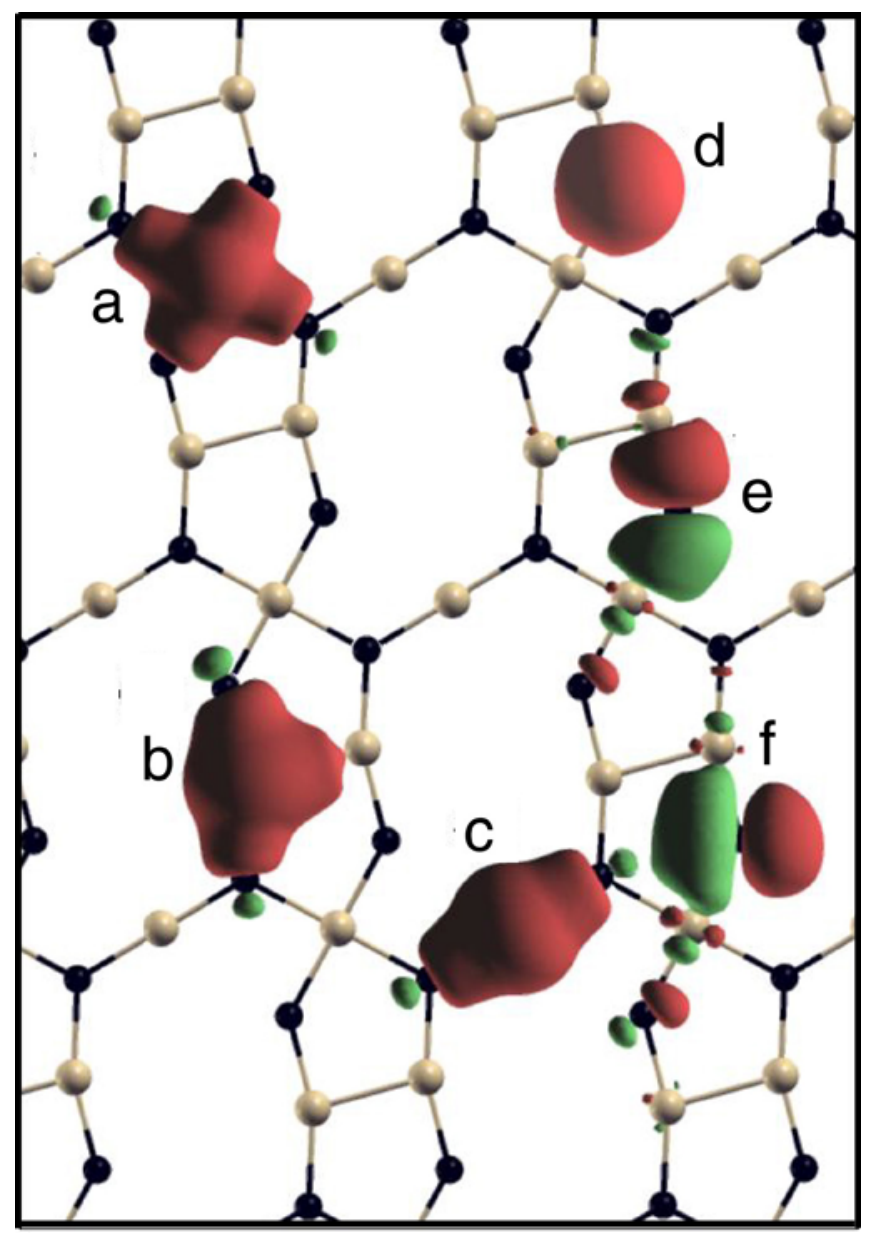

FIG. 5. (color online) AOs in structure $\boldsymbol{A}$. $6 s \mathrm{AOs}$ for (a) $\mathrm{Au}_{(4)}$, (b) $\mathrm{Au}_{(3, \mathrm{a})}($ similar to $\left.\mathrm{Au}_{(3, \mathrm{~b})}\right)$, (c) $\mathrm{Au}_{(2)}$; (d) $3 s \mathrm{AOs,} \mathrm{(e)} \mathrm{and} \mathrm{(f)} 3 p$ AOs for $\mathrm{S}_{(2, \mathrm{~b})}$ (similar to other $\mathrm{S}$ atoms). Red and green surfaces represent positive and negative contour surfaces of the same absolute value. 


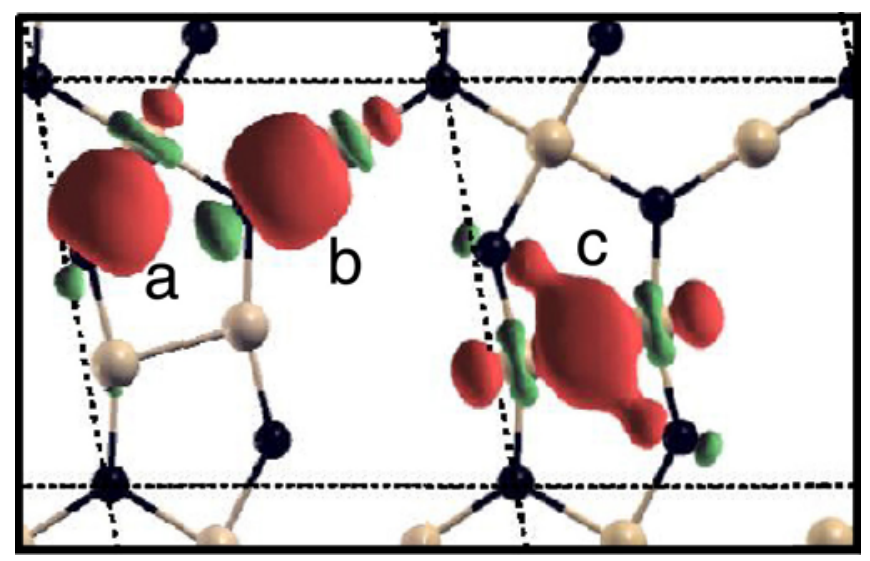

FIG. 6. (color online) BOs in structure $\boldsymbol{A}$. (a) $\mathrm{Au}_{(4)}-\mathrm{S}$, (b) $\mathrm{Au}_{(2)}-\mathrm{S}$, (similar to $\left.\mathrm{Au}_{(3, \mathrm{a})}\right)^{-} \mathrm{S}$ and $\left.\mathrm{Au}_{(3, \mathrm{~b})^{-}} \mathrm{S}\right)$ and (c) $\mathrm{Au}_{(3, \mathrm{a})^{-}} \mathrm{Au}_{(3, \mathrm{~b})}$. Symbols are the same as in Fig. 5. 


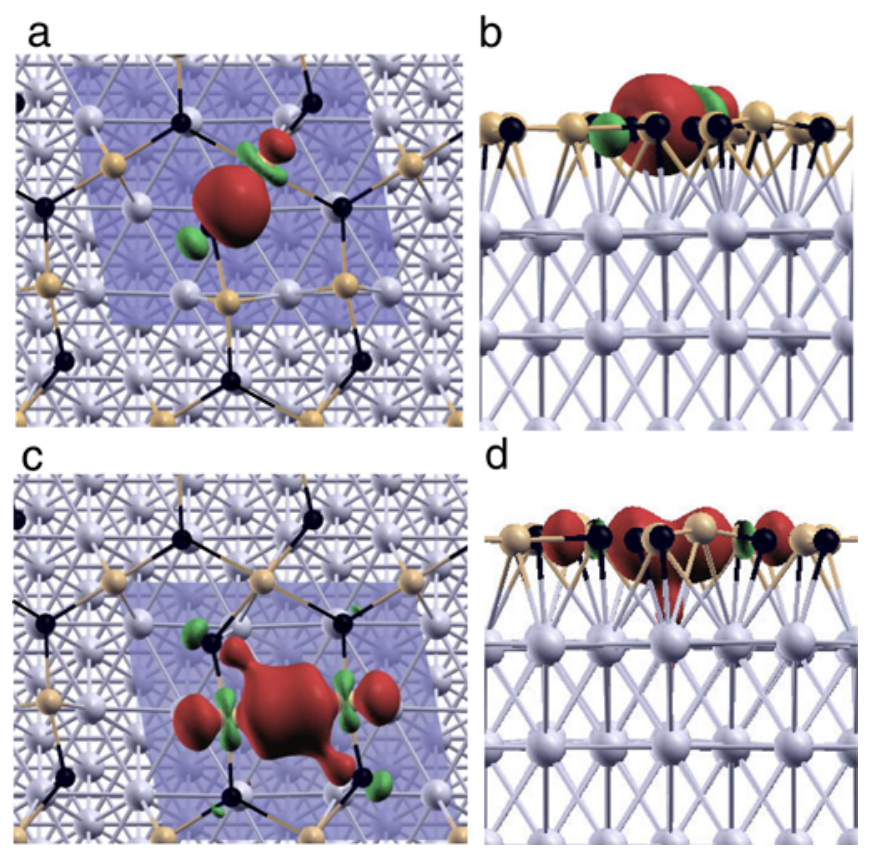

FIG. 7. (color online) BOs in structure $B \cdot \mathrm{Au}_{(2)}-\mathrm{S}$ in (a) top and (b) side views (similar for other $\mathrm{Au}-\mathrm{S}$ bonds), and $\mathrm{Au}_{(3, \mathrm{a})}-\mathrm{Au}_{(3, \mathrm{~b})}$ in (c) top and (d) side views. Symbols are the same as in Fig. 5. The isocontour value in (c) is half that in (a), (b) and (d). 